\title{
A Search for Variable Stars at the Galactic Center
}

\author{
JOSEPH W. HALLER \\ MARCIA J. RIEKE \\ Steward Observatory \\ University of Arizona \\ Tucson, AZ 85721
}

\begin{abstract}
A study of the Galactic Center stellar population is continuing with a sensitive $2 \mu \mathrm{m}$ CCD camera. Using a $64 \times 64$ detector array, background limited images are recorded with modest amounts of observing time $\left(t_{\text {obs }} \approx 20\right.$ sec to reach $K=13$ ). Magnitudes have been extracted using DAOPHOT from repeated imaging of the central $5^{\prime} \times 5^{\prime}$ to search among approximately 1500 stars for long period variables (LPV's, $P>200^{d}$ ), particularily Miras. Miras have a well defined periodluminosity relationship as well as one in period-mass. This program investigates the nature of highly luminous stars at the Galactic Center. Presently 12 variables have been found and have several characteristics consistent with Miras. They have a maximum bolometric luminosity of $-4.4 \mathrm{mag}$, which supports the case that high luminosity stars in the central $6 \mathrm{pc}$ are young supergiants.
\end{abstract}

\section{Introduction}

Previous work (Rieke 1986; Rieke 1988) based on $2 \mu \mathrm{m}$ mapping has investigated the luminosity distribution of the stars in the central $5^{\prime} \times 5^{\prime}$ region at the Galactic Center. When the bolometric magnitudes are compared with the distribution in Baade's window it is apparent that the Galactic Center contains a large number of stars up to 3 magnitudes brighter than the luminosity cutoff in the bulge. The question then arises: What is the nature of this high luminosity population? Is this population the tail of a distribution similar to Baade's window? Alternatively it could be a younger supergiant population implying a period of recent star formation. Rieke (1988) argued for the latter case by showing that if a luminosity distribution like Baade's window applied to the Galactic Center, the excess tail is highly unlikely to be due to simply increased numbers or to foreground bulge stars, and thus they represent a younger population. If they are the high luminosity tail of a bulge distribution, then they will be AGB stars and many will exhibit the characteristic Mira pulsation. For a stellar population of a given age, the most massive stars on the AGB will have the highest average luminosity and longer period. Thus locating the average brightness of the Miras yields a rough estimate of the age of the stellar population. Alternatively, if there is a younger supergiant population present with an older population it would be expected that Miras would be found at a magnitude near the peak of the Baade's window population. To an- 
swer the question one must search for variables and determine where they occur in the bolometric luminosity function.

\section{Observations}

A long term program was begun in May 1986 to repeatedly image the central $5^{\prime} \times 5^{\prime}$ region of the Galactic Center. Observations were performed on the Steward $1.54 \mathrm{~m}$ telescope using a $2 \mu \mathrm{m}$ camera with a $64 \times 64$ detector array with 1.2 arcsec pixels. A particular map is made of 7 columns with 5 to 9 rows each, totaling approximately 50 frames. Also each source was imaged at least twice at a given epoch due to frame overlap. Maps in the current analysis were made in May 1987, September 1987, and May 1988 with additional data available from a map made in May 1986.

Each frame was exposed for 20 seconds, bad pixels removed, and flatfielded and reach a $3 \sigma$ limiting magnitude of $K=13$. Further reductions were performed using the programs IRAF and DAOPHOT (Stetson, 1986). To determine a detection threshold an estimate of the standard error per pixel was needed. This was derived empirically on selected regions of frames distributed across the map which appeared free from any obvious sources. This showed that the $\sigma_{\text {photons }} \approx 0.1 \times \sigma_{\text {total }}$, so that the dominant source of noise is the faint unresolved stars and diffuse background. For each frame a point spread function was constructed from a candidate list of the 20 brightest sources. The stars were then grouped and their magnitudes and positions were determined by the multiprofile fitting subroutine NSTAR in DAOPHOT.

As a preliminary to full scale reductions a 4.5 square arcminute area representing about $20 \%$ of the region was examined to a limiting $K$ magnitude of 12 for the May and September 1987 maps. The magnitude of a given star was then determined to be repeatable to better than $0.1 \mathrm{mag}$ at a given time. If any star had a variation $|\Delta K|>0.2 \mathrm{mag}$ between the two maps it was deemed variable. Further reductions will include all stars. Maps have also been made at $H$ and $L$ to extract color information.

\section{Results}

Spectroscopic evidence shows that most of the stars in this region are of late $M$ spectral type (Rieke and Rieke 1988; Rieke 1988). Figure 1 shows a representative sample of $2.2 \mu \mathrm{m}$ spectra taken of some of the more luminous stars in the field. Also shown are two spectra from Baade's window, one of M5III and the other of type M9III. As can be seen the luminous stars at the Galactic Center show the same general features, namely, the $\mathrm{H}_{2} \mathrm{O}$ absorption and $\mathrm{CO}$ band heads. Within 
this range of spectral types the bolometric corrections applied to $\mathrm{K}$ magnitudes are nearly constant (Frogel and Whitford 1987; G. Rieke, personal communication). Also a constant $K$ extinction of 3.5 was assumed based on the $H$ and $K$ maps from May, 1986 which indicate roughly constant extinction outside of a few regions where the extinction is much higher. A distance to the Galactic Center of $8.0 \mathrm{kpc}$ was assumed to then obtain the absolute bolometric magnitudes. Of the 154 stars surveyed, 12 had varied between May and September 1987. The average magnitudes and amplitude of the variations for these stars are shown in table 1. A bolometric luminosity distribution of the variables is then compared with the distribution of the entire sample in figure 2. The magnitudes listed are uncertain up to $0.5 \mathrm{mag}$, mainly due to the variable extinction across the surveyed area.

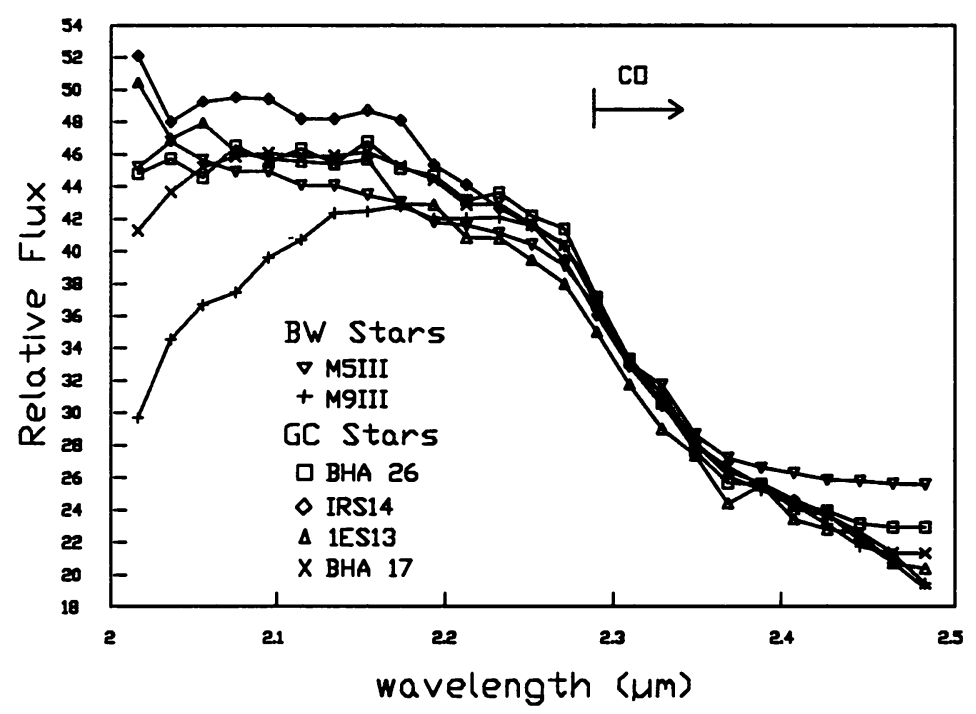

Figure 1. A representative sample of $2.2 \mu \mathrm{m}$ spectra of Galactic Center stars. Also shown are spectra from a M5III and a M9III from Baade's window. Spectra are arranged so as to match the $\mathrm{CO}$ band head, and are consistent with being late $\mathrm{M}$. From Rieke and Rieke 1988, work in progress. 


\begin{tabular}{|c|c|c|c|}
\hline & $\underline{\overline{\mathbf{K}}}$ & $\begin{array}{l}|\Delta K| \\
\mid \Delta K\end{array}$ & $\mathrm{M}_{\text {bol }}$ \\
\hline 1 & 10.45 & 0.30 & -4.38 \\
\hline 2 & 10.50 & 1.00 & -4.33 \\
\hline 3 & 10.56 & 0.32 & -4.27 \\
\hline 4 & 10.58 & 0.45 & -4.25 \\
\hline 5 & 10.68 & 0.35 & -4.15 \\
\hline 6 & 10.78 & 0.35 & -4.05 \\
\hline 7 & 11.12 & 0.23 & -3.71 \\
\hline 8 & 11.25 & 0.23 & -3.58 \\
\hline 9 & 11.39 & 0.31 & -3.45 \\
\hline 10 & 11.64 & 0.32 & -3.19 \\
\hline 11 & 11.77 & 0.33 & -3.06 \\
\hline 12 & 11.95 & 0.30 & -2.88 \\
\hline
\end{tabular}

Table 1. The average $K$ magnitude and absolute value of the variation between May and September 1987. Also listed is the bolometric magnitude with an assumed extinction at $\mathrm{K}$ of 3.5, a bolometric correction of 3.16, and a Galactic Center distance of $8.0 \mathrm{kpc}$.

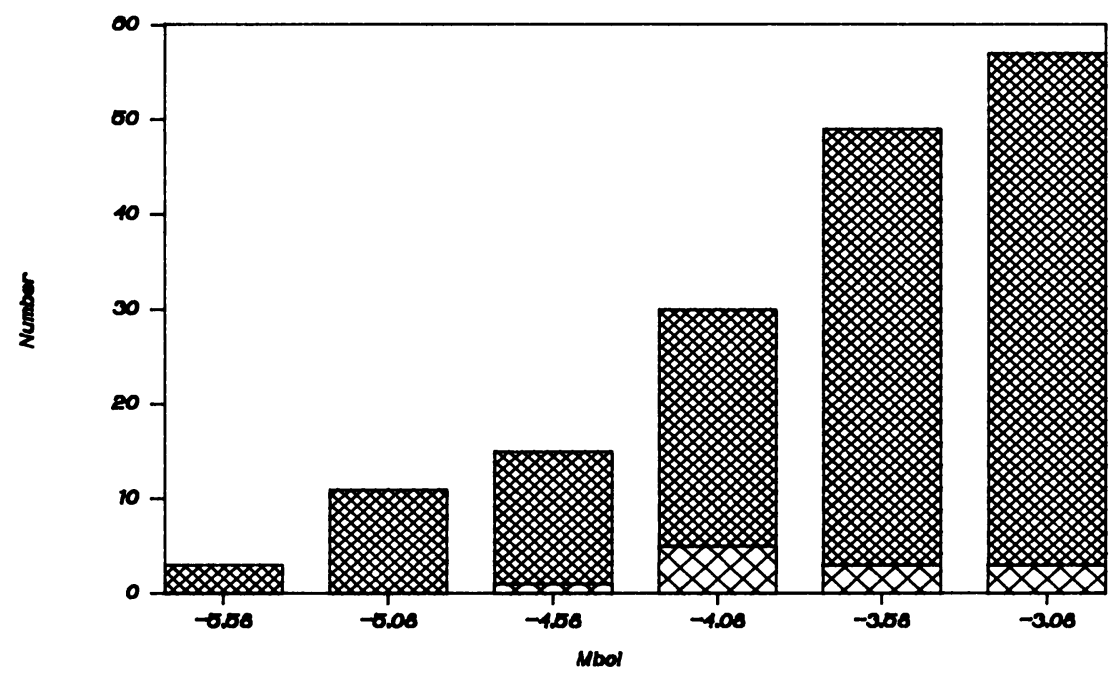

Figure 2. The derived luminosity function from the surveyed area using a bin width of $0.5 \mathrm{mag}$. Coarser hatching indicates the distribution of variables while finer hatching indicates the total number of stars in the magnitude interval. Note the cutoff in the distribution of variables at $\mathrm{M}_{\mathrm{bol}} \approx-4.5 \mathrm{mag}$. 


\section{Discussion}

Several things point to identifying these stars as Miras. Their amplitudes and the timescale of their variations are quite characteristic of Miras (Feast et al. 1982). Also when one accounts for the extinction one finds that they have apparent $\mathrm{K}$ magnitudes similar to the Miras observed through Baade's window (Glass and Feast 1982). Interestingly if these are all indeed Miras they have a number density on the sky 50 times greater than what is observed in the bulge. Thus it is highly unlikely that some are foreground stars and indeed are at the Galactic Center. If the current rate of detection continues, on the order of 100 variables may be found which will provide a large sample for statistical studies.

There is preponderance of variables at the low luminosity end and none at the high end. Not only that, but within each magnitude interval, the number of variables never dominates. Most importantly the cut off in the variable star distribution matches closely with that in Baade's window which occurs at $\mathbf{- 4 . 2}$ (Frogel and Whitford 1987) It would then seem likely that these Miras could be identified with the peak of the older bulge distribution of stars. This then supports the interpretation that the large number of luminous late $M$ stars are young supergiants evolved from the high mass end of the main sequence. This would then imply that a recent period of star formation occured approximatly $10^{8}$ years ago which created the preponderance of luminous stars seen. In other words, clarification of the nature of these luminous stars is made by saying what they are not (i.e. They are not late type giants in the Mira phase at the tip of the AGB.). Obviously these conclusions are tentative because only a small fraction of the area has been analyzed.

Further work will include confirming the Mira characteristics on the basis of H-K,K-L color diagrams which can then be use to identify Miras further away from the center without having to go through the laborious process of searching for magnitude variations. If they behave similarly to Miras in the rest of the Galaxy and the LMC they could be used as distance indicators to the Galactic Center or assuming they do indeed act as standard candles they could be used to map the extinction across the region.

\section{Conclusions}

A background limited survey for LPV's to $K=13$ is in progress for the central $5^{\prime} \times 5^{\prime}$ of the Galactic Center. Preliminary analysis for a 4.2 square arcminute field shows that they occur with a high number density and have $\mathrm{K}$ magnitudes and amplitudes consistant with being identified as Mira variables and associated with the bulge population. By implication the high luminosity tail of the luminosity distribution is composed of young supergiants. This supports the case for a starburst in the region $10^{8}$ years ago. A more complete analysis is in progress for the entire 25 square arcminute field.

We would like to acknowledge the useful discussions with G. Rieke, R. Catch- 
pole, J. Frogel, M. Feast, and E. Olszewski. A good deal of thanks goes to I. Barg in setting up the data reduction facilities.

\section{References}

Feast, M.W. ,Robertson, B.S.C., Catchpole, R.M., Lloyd Evans, T., Glass, I.S., and Garter, B.S., M.N.R.A.S., 1982, 201, 439

Frogel, J.A., and Whitford, A.E., Ap.J., 1987, 320, 199

Glass, I.S., and Feast, M.W., M.N.R.A.S., 1982, 198, 199

Rieke, M.J., Nearly Normal Galaxies, 1986, 90

Rieke, M.J., Galactic and Extragalactic Star Formation, 1988, 345

Rieke, G.H., and Rieke, M.J., Ap. J., 1988, 330, L33

Rieke, G.H., and Rieke, M.J., 1988, In progress

Stetson, P.B., Pub. Astron. Soc. Pac., 1986, 99, 191 